\title{
Novel fiber-optical accelerometer for monitoring tasks of power generators
}

\author{
Michael Villnow, Siemens AG, Corporate Technology, Erlangen, Germany \\ Dr. Thomas Bosselmann, Siemens AG, Corporate Technology, Erlangen, Germany \\ Dr. Michael Willsch, Siemens AG, Corporate Technology, Erlangen, Germany \\ Prof. Bernhard Schmauss, Lehrstuhl für Hochfrequenztechnik, University Erlangen-Nuremberg, Erlangen, \\ Germany
}

\section{Introduction}

Due to the increase in energy demand and the concept of sustainable engineering, modernizations of electric power plant installations become more and more important for two reasons: first to improve the energy efficiency and second to save costs and materials. However, the operating point of electric machines like power generators gets closer to its physical limit. To prevent damages and to verify optimization measures, sensors and monitoring systems are particularly essential. Therefore a novel fiber-optical acceleration sensor has been developed. In this paper the sensor's principle of operation is explained, laboratory tests are shown, the sensor behavior is characterized and real life measurements are presented.

\section{Function Principle}

The Siemens Fiber Optical Accelerometer (SFOA) is based on the intensity modulation of light. The end surface of a bare multimode fiber is placed towards a tilted mirror. Thereby the fiber is mounted into a fiber ferrule and fixed by a solid adhesive bond. A defined length $l$ of the fiber sticks out at the end of the ferrule like a cantilever bar, as depicted in Figure 1a).

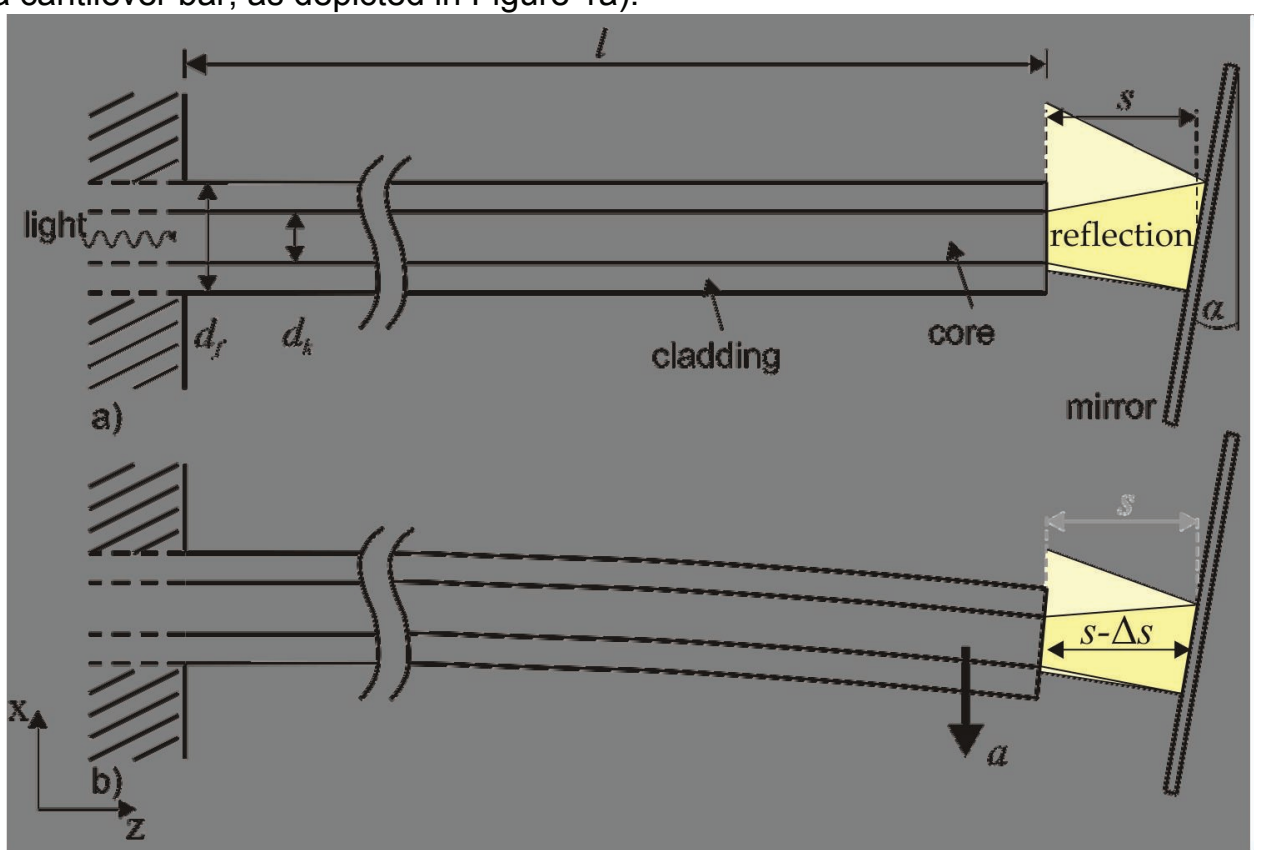

Fig. 1: working principle of the Siemens Fiber Optical Accelerometer (SFOA)

When light is launched into the fiber the light leaves the waveguide at its end surface, is reflected by the mirror and partially coupled back into the fiber. Due to the aperture of the fiber, the light beam is radiated conically divergent, thus only a small percentage of the reflected light is coupled back into the fiber. When the sensor head is exposed to an acceleration $a$, as depicted in figure $1 \mathrm{~b}$ ), the protruding fiber end carries out a relative motion with respect to the mirror. In consequence of the geometric configuration of the tilted mirror, the distance between fiber end surface and mirror changes due to the motion. Based on these distance changes the optical path of the conical light beam gets shorter and the reflected light beam is less widened. Hence more light is coupled back into the fiber. When the fiber is deflected in the opposite direction, the distance between fiber end surface and mirror increases, whereby less light is coupled back 
into the core of the fiber. As a result the intensity I of the reflected and coupled light is modulated according to the occurring acceleration. To characterize the performance and sensitivity of the sensor, the modulation ratio $M_{\mathrm{r}}$ (henceforth just called modulation) has to be calculated, taking into account the background intensity $I_{\text {ave }}$ and the measured acceleration $a$, as shown in equation 1.1.

$$
M_{\mathrm{r}}=\frac{I-I_{\mathrm{avc}}}{I_{\mathrm{avc}} \cdot a}=\frac{\Delta I}{I_{\mathrm{avc}} \cdot a}
$$

\section{Resonance Frequency}

Due to the protruding length of the fiber $l$, its diameter $d_{\mathrm{f}}$, the density $\rho$ and the specific E-modulus E, the resonance frequency of the sensor head is set to a specific frequency. By setting up the balance of forces and moments of a fiber surface element, the resonance frequencies of the protruding fiber can be calculated. Equation 1.2 shows the correlation between the resonance frequency $f_{\text {res }}$ of the protruding fiber and its physical parameters in good approximation.

$$
f_{\mathrm{r}} \sim \frac{d_{\mathrm{f}} \cdot \sqrt{\mathrm{E}}}{l^{2} \cdot \sqrt{\rho}}
$$

With regard to using the SFOA to measure vibrations on the end windings of a power generator, the resonance frequency of the sensor should be set to a value, which is far enough away from the measurement frequency. Normally a generator runs with a nominal frequency of $50 \mathrm{~Hz}$, in some parts of the world with a nominal frequency of $60 \mathrm{~Hz}$ e.g. in the U.S.. Due to the alternating magnetic field the induced vibrations, mainly caused by magnetic forces, show a frequency twice the nominal frequency, thus up to $120 \mathrm{~Hz}$. To calculate a modal analysis of a vibration mode, the sensor should be able to measure at least the second harmonic. In consequence the resonance frequency of the SFOA has to be higher than $240 \mathrm{~Hz}$, or even more than $300 \mathrm{~Hz}$ to ensure an adequate margin to separate vibration frequencies from the sensor's resonance frequency.

\section{Sensitivity}

Normally the vibrations occurring in an electric engine should not exceed the acceleration limit of $10 \mathrm{~m} / \mathrm{s}^{2}$, corresponding to a displacement of 50 micrometers peak-to-peak at $100 \mathrm{~Hz}$. In some cases the accelerations at some locations can be much higher. Hence the objective target is to realize a sensor that can measure accelerations over a wide range of about $100 \mathrm{~m} / \mathrm{s}^{2}$ having a resolution of $1 \mathrm{~m} / \mathrm{s}^{2}$ or $0.1 \mathrm{~g}$.

The sensitivity of the SFOA is defined by the movement of the fiber end placed towards the mirror. The more the fiber end is displaced the more the light intensity is modulated due to distance changes between the fiber end surface and the mirror. Equation 1.3 shows the dependency of the fiber deflection $\Delta x$ at its end.

$$
\Delta x=\frac{q \cdot l^{4}}{8 \cdot \mathrm{E} \cdot \mathrm{I}}
$$

Here $q$ is the line load caused by the fiber's mass and I is the area moment of inertia. The area moment of inertia is given by the equation 1.4 according to a symmetric circular surface.

$$
\mathrm{I}=\frac{\pi \cdot d_{\mathrm{r}}^{4}}{64}
$$

It can be recognized that the deflection depends on some material constants and mainly on the protruding fiber length $l$. To achieve a higher deflection and therefore a higher modulation of light intensity, the protruding fiber length has to be elongated. However this requirement contradicts to the behavior of the sensor's resonance frequency, which limits the length of the fiber. Finally a trade-off between the sensitivity of the sensor head and the resonance frequency has to be made. As a result the resonance frequency is set to $350 \mathrm{~Hz}$, which shows a sufficient distance to the second harmonic of most electric machines. The lateral deflection at the fiber end is less than 1 micron per $\mathrm{m} / \mathrm{s}^{2}$, but the resulting modulation is still about $0.001 \mathrm{~s}^{2} / \mathrm{m}$.

\section{Directional sensitivity}

Information about the direction of occurring forces is very important for the design of electric engines and the dimensioning of support elements. To measure the direction of e.g. end winding vibrations, the acceleration sensor has to be directional sensitive. This is achieved by the geometric arrangement of the mirror. Figure 2 shows a simplified drawing of the sensor head with two different views. Figure 2a) shows the side view, also shown before in figure 1 . Figure $2 b$ ) gives a top view on the geometric configuration of the sensor head. 


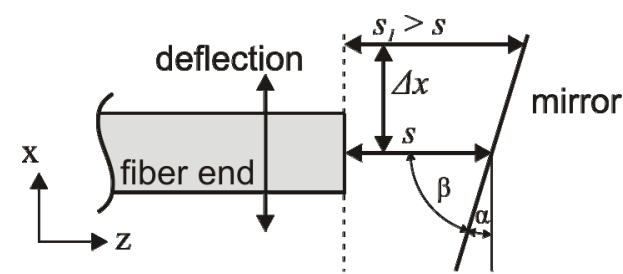

a) side view

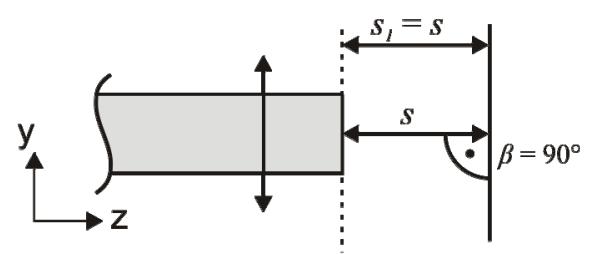

b) top view

Fig. 2: simplified geometric sensor head configuration from two different views

By deflecting the fiber in the x-z-plane the distance between fiber end surface and mirror changes $\left(s_{1}>s\right)$ leading to a light modulation. When the fiber performs a movement in the $y$-z-plane, no significant distance changes between fiber end surface and mirror occur $\left(s_{1}=s\right)$. The reason for this is very simple, based on the elementary trigonometric functions. The distance change $\Delta z=s_{1}-s$ can be calculated by the tangent function, with the tilted angle of the mirror $\alpha$ and the deflection $\Delta x$, shown in equation 1.5.

$$
\Delta z=\Delta x \cdot \tan (\alpha)
$$

Since the mirror is not tilted in the $y$-z-plane, the angle of the mirror is zero $\left(\alpha=0^{\circ}\right)$. Consequently the distance change also returns zero $(\Delta z=0)$ by equation 1.5 . This geometric correlation is illustrated in figure $2 b$ ).

Beside this distance chance the fiber gets bent due to acceleration forces and the fiber's mass, so the fiber end surface tilts during the deflection. The bending angle $\varphi$ at the end of the protruding fiber can be calculated by equation 1.6 .

$$
\varphi(l)=\frac{q \cdot l^{3}}{6 \cdot \mathrm{E} \cdot \mathrm{I}}
$$

With a typical acceleration of $a=10 \mathrm{~m} / \mathrm{s}^{2}$ the bending angle of the fiber end surface results to $\varphi(l) \approx$ $0.00025^{\circ}$, which is less than one angular second. As a consequence the influence of the beam distortion caused by fiber bending can easily be ignored for accelerations of up to $100 \mathrm{~m} / \mathrm{s}^{2}$. Considering all geometric correlations and simplifications the sensor head is in very good approximation (error less than $2 \%$ ) only sensitive for deflections in the x-direction.

\section{Sensor design and materials}

The sensor head consists of non-metallic materials like GRP (glass fiber reinforced plastic), Teflon, ceramic and epoxy resin. Thus this type of sensor can be used for measurements under harsh conditions, e.g. high temperatures of up to $150^{\circ} \mathrm{C}$ and strong magnetic fields that prevail in power generators. Figure 3 shows a picture of the sensor head and several components of the inner body.
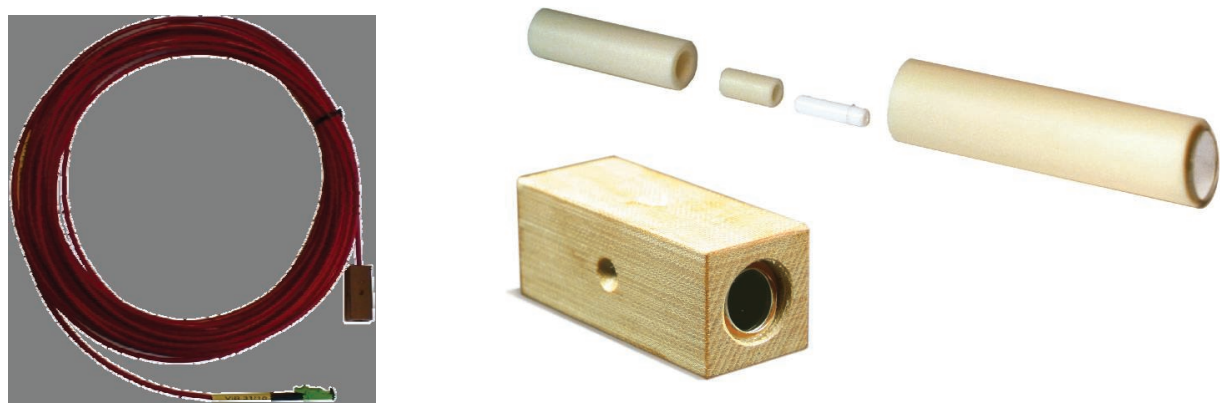

Fig. 3: Sensor housing and sensor head elements

The housing of the sensor is made of laminated fabric, so it is well insulated and applicable even for high voltage tasks. The housing has a quadratic cross sectional area, which defines the sensitive and nonsensitive axes of the sensor head. The inner sensor body is built from ceramic tubes with different diameters. All tubes fit perfectly together with little tolerances, so the sensor can be built by modular elements. The mirror is mounted on the end surface of the outer ceramic tube. To bond all parts of the sensor head, a heat curing epoxy resin glue is used.

The cable length of the sensor can be customized to suit specific needs. The fiber is protected by a dimensionally stable Teflon tube. So the sensor can easily be used even under rough conditions e.g. in 
power plant installations. Another advantage is the thermal resistance of Teflon of up to $220^{\circ} \mathrm{C}$, so the cable can be placed even along machine hot spots. An E-2000 angled fiber connector with a dust cover is used to protect the connector's fiber surface.

\section{Interrogation unit and signal processing}

A modular interrogation unit was developed to read out the fiber optic accelerometer. Every single measuring channel is built on its own circuit board, realized as a slide-in card for a 19" rack unit. All in all 16 channels can be run by one single interrogation unit. The latest version is shown in figure 4a).
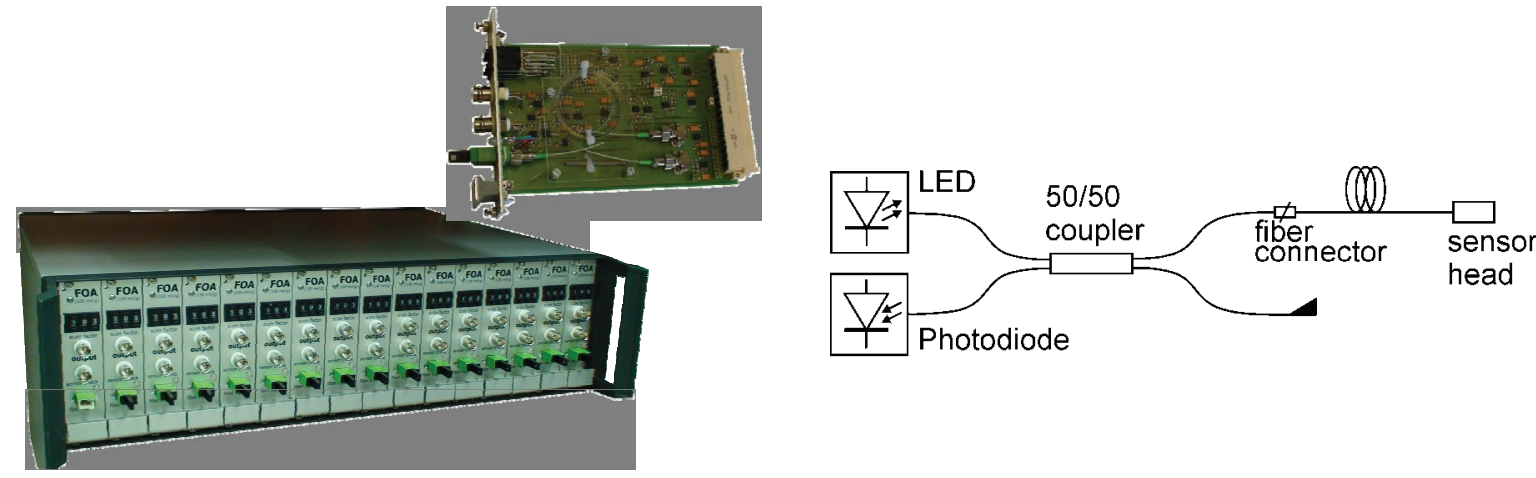

Fig. 4: a) Interrogation unit with 16 slide-in sensor cards

b) Block diagram of sensor signal light path

To illuminate the sensor head, a LED with a center wavelength of $820 \mathrm{~nm}$ and a spectral width of $50 \mathrm{~nm}$ is used. The LED light is coupled into a multimode glass fiber and guided by a $3 \mathrm{~dB}$ fiber coupler to a connector at the front plate, where the sensor can be plugged in. The modulated light, coming back from the sensor head, is guided to a photodiode by passing the $3 \mathrm{~dB}$ fiber coupler, as shown in figure $4 \mathrm{~b}$ ).

The photo current is converted to a voltage signal by a transimpedance amplifier. To guarantee an ideal sensor operating point, the light intensity is regulated by a controller which attunes the LED current to all attenuation influences along the light path. Hence the sensor can still be used even if its cable is deformed or bent around edges with small curve radii. The voltage signal of the sensor can now be processed by different filter and normalization options. To suppress the resonance frequency of the sensor a low-pass filter can be activated. To eliminate the fluctuations in sensitivity of each sensor head caused by production tolerances, a level adaption is realized so every sensor shows the same behavior and signal output. In addition a normalization is implemented to calibrate the sensor signal to a constant factor of $10 \mathrm{mV} /\left(\mathrm{m} / \mathrm{s}^{2}\right)$. The edited signal can now be recorded by an external transient recorder or an oscilloscope.

However, this fiber optic acceleration sensor shows a frequency-dependent behavior, so it is necessary to carry out a mathematical post-processing when the exact vibration amplitude of signals with superposed vibrations at different frequencies is needed. The frequency response of a representative sensor, calibrated to $10 \mathrm{mV} /\left(\mathrm{m} / \mathrm{s}^{2}\right)$ for $100 \mathrm{~Hz}$, is shown in figure 5 .

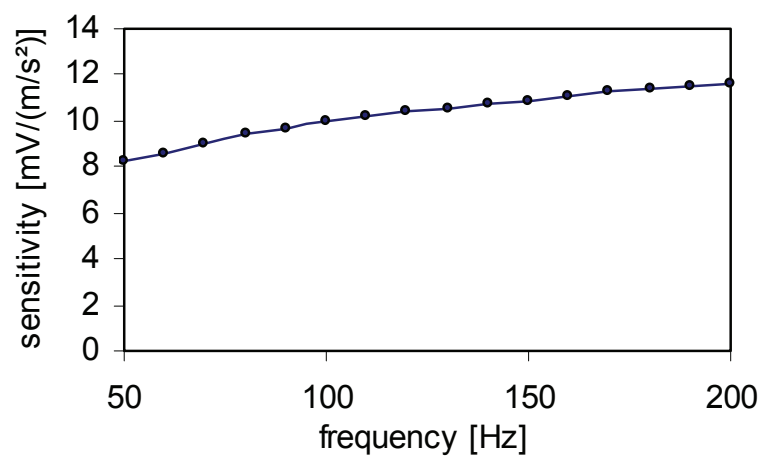

Fig. 5: Frequency response of the fiber optic accelerometer

A recorded transient signal can be separated into its frequency components by a Fourier transform. In the frequency domain an amplitude adaption can be carried out corresponding to the inverse frequency responce of the sensor. Finally the signal can be transformed back to the time domain which results in a corrected vibration amplitude. As an example a typical vibration signal is shown in figure 6a), its corresponding Fourier transformed signal is shown in $6 \mathrm{~b}$ ) and the corrected time domain signal in 6c). 

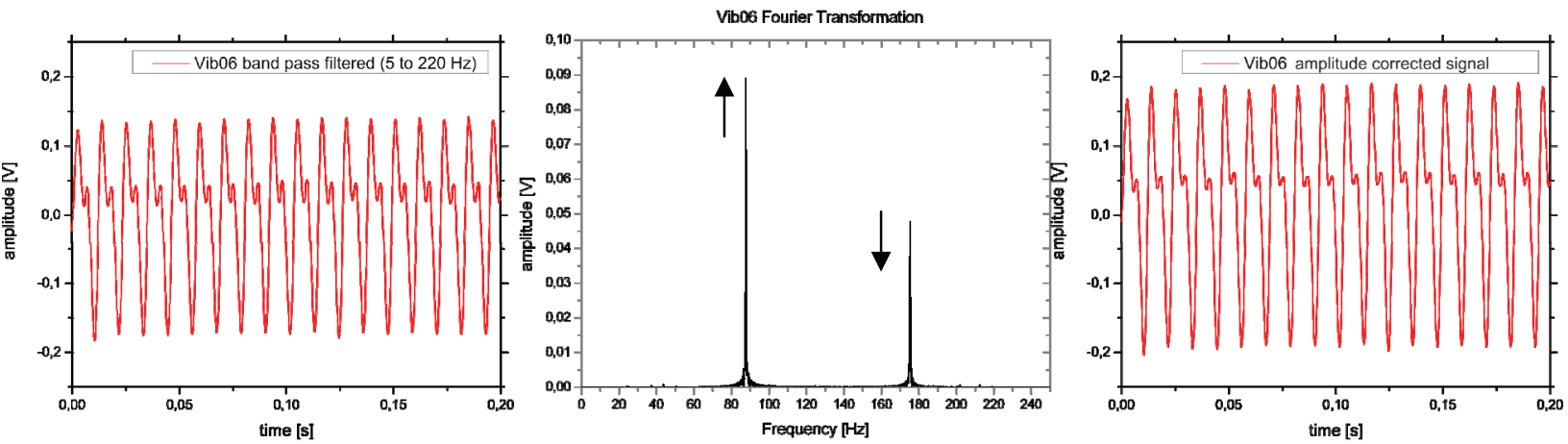

Fig. 6: a) Typical transient signal; b) Fourier transform signal; c) Corrected time domain signal

\section{Field measurement results}

Since 2009 several field tests have been performed to demonstrate the sensor functionality under real life conditions, to prove the thermal robustness and magnetic ruggedness of the sensor and finally to open up new fields of application. In 2009 the first Siemens Fiber Optical Accelerometers have been placed at the end winding connectors of a 300 MVA hydro-powered motor-generator of a water pump storage power plant. Fourteen sensors have been mounted on seven different measuring points, measuring the tangential and radial vibration components. The measurements have been performed at different generator machine conditions, like short circuit, open circuit, base load and over excitation. During all measurements no sensor failed or caused any type of problems like partial discharge.
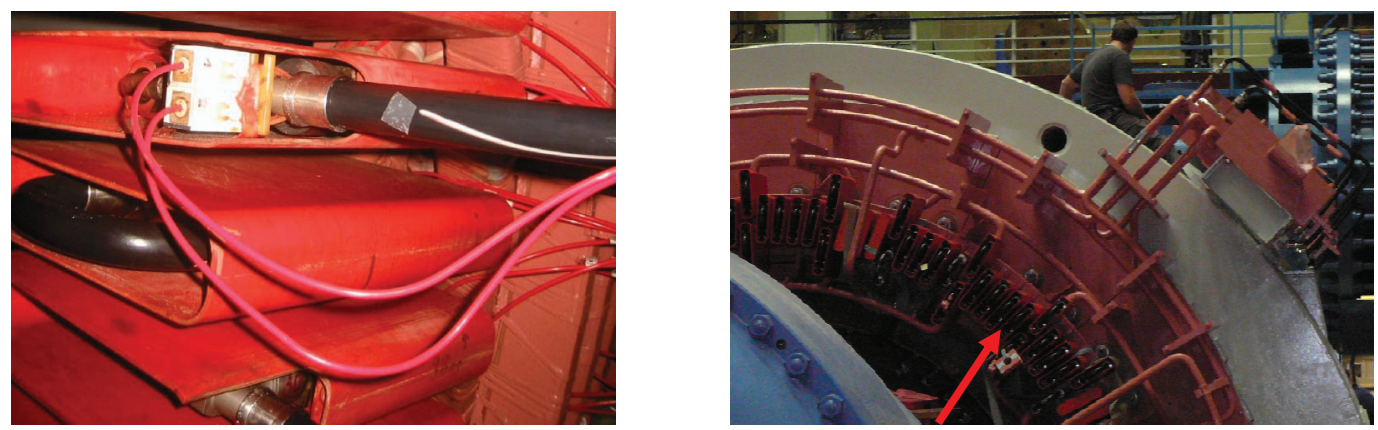

Fig 7: SFOAs mounted on the end winding connector of a 300 MVA generator

While recording all sensor signals during a machine run-up, the frequency and the amplitude of the machine's resonance peaks can be calculated. The according measurement is shown in figure 8a). As a consequence the influence of enhanced resonance vibrations on the durability of the machine can be estimated. The operator of a power plant is now able to avoid operating points with increased vibration amplitudes to improve the lifetime of the machine.

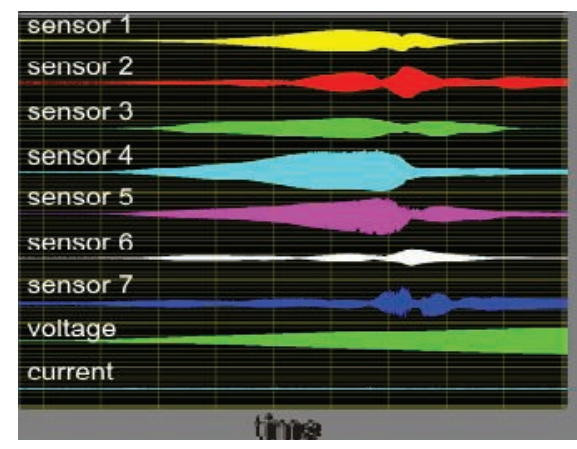

Fig 8: a) Machine run-up, open circuit

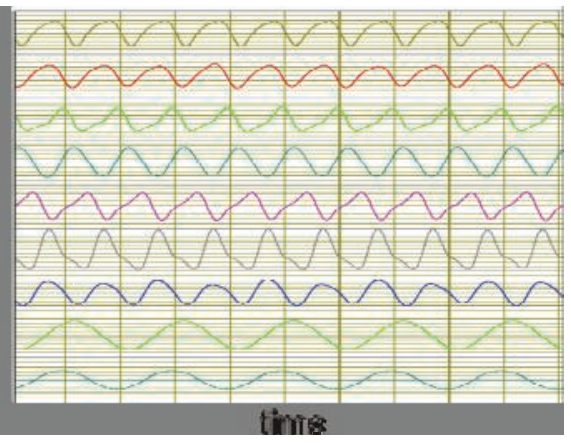

b) End winding vibrations, base load/over excitation

By using fiber optic vibration sensors directly placed on highly stressed machine parts like stator bars or supporting elements, small damages can be detected early so that machine failure or a complete destruction can be prevented. 
In figure 8b) the vibration amplitude of seven sensors is shown, while the generator is running in base load with an over excited rotor. At this operating point the highest acceleration amplitudes have been measured, rising up to $25 \mathrm{~m} / \mathrm{s}^{2}$ and more. Looking at the shape of the measured vibration curve shown in figure $8 \mathrm{~b}$ ), it can be realized that the machine does not only oscillate at its fundamental mode but at additional higher modes.

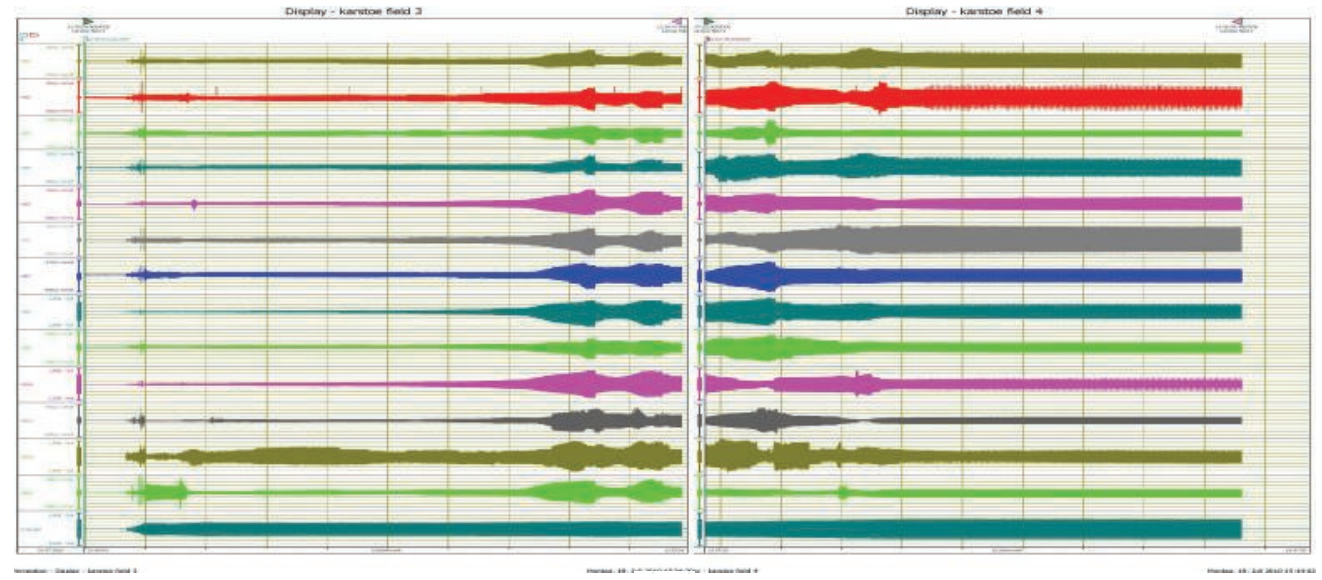

Fig. 9: End winding vibrations during machine run-up of a $40 \mathrm{MVA}$ compressor motor of a gas transfer and distribution station

A further field test was made during the run-up of a completely renewed 40 MVA compressor motor. There the sensors had to work in an explosion protected area. Therefore a special feedthrough was constructed to guarantee the gas-proofed sealing. The recorded vibration amplitudes measured by fifteen SFOAs are shown in figure 9. It has been recognized that this machine has a lot of increasing resonances at different constellations of rotating frequency and compression output power. However, the maximum vibration amplitude never exceeded a value of more than $20 \mathrm{~m} / \mathrm{s}^{2}$.

\section{Conclusion}

A novel fiber optic accelerometer called SFOA has been developed and investigated. The working principle is based on the modulation of light using a simple configuration of only one fiber and one mirror. No moving parts are necessary which leads to a very tough but also small sensor housing. Due to the choice of special non-metallic materials the sensor can be used under harsh conditions like magnetic fields, high voltage or temperatures of up to $150^{\circ} \mathrm{C}$. Because of a passive operating sensor concept using a low power LED, the accelerometer can even be used in an explosion protected area without further precautions. Furthermore, the sensor can be used for monitoring tasks of high accelerations e.g. sudden short circuit vibrations of up to $700 \mathrm{~m} / \mathrm{s}^{2}$ by changing the sensor dimensioning.

By calculating the mechanical behavior of the sensor head, an optimized configuration was found to keep the resonance frequency and the sensitivity high. Several prototypes have been built to eliminate production errors and to characterize residual production tolerances. To the present day several field measurements have been made to prove the durability and to test the sensor behavior under real life conditions. As a result of these successful measurement campaigns, the complete sensor system turned out to be a useful enlargement of existing monitoring systems to prevent damages and machine failures.

[1] R. O. Cook and C. W. Hamm, "Fiber optic lever displacement transducer", Applied Optics,Vol. 18, Issue 19, pp. 3230-3241, 1979

[2] J. Kalenik, R. Pajak, "A cantilever optical-fiber accelerometer", Sensors and Actuators A: Physical, Volume 68, Issues 1-3, Pages 350-355, EurosensorsXI, 1998

[3] K.-H. Grote and J. Feldhusen, „Dubbel - Taschenbuch für den Maschinenbau“, 22. Aufl. Springer Berlin Heidelberg New York, 2007

[4] G. Conforti, M. Brenci, A. Mencaglia, and A. G. Mignani, "Fiber optic vibration sensor for remote monitoring in high power electric machines," Appl. Opt., vol. 28, no. 23, pp. 5158-5161, Dec. 1, 1989.

[5] J.M. López-Higuera et al., "Simple low frequency optical fiber accelerometer", Journal of Lightwave Technology, VOL. 15, NO. 7, July 1997 